\title{
Outcomes of Retreatment for Intracranial Aneurysms - A Meta-Analysis
}

\author{
Ivo S. Muskens, MD*‡§ \\ Omar Hertgers, MD* \\ Geert J. Lycklama à Nijeholt, \\ MD, PhD" \\ Marike L.D. Broekman, MD, \\ PhD, LLM*‡\| \\ Wouter A. Moojen, MD, PhD, \\ MPH $^{* \# \#}$
}

*Department of Neurosurgery, Haaglanden Medical Center, The Hague, The Netherlands; ${ }^{\ddagger}$ Department of Neurosurgery, Leiden University Medical Center, Leiden, The Netherlands; ${ }^{\S}$ Center for Genetic Epidemiology, Department of Preventative Medicine, Keck School of Medicine, University of Southern California, Los Angeles, California; "Department of Radiology, Haaglanden Medical Center, The Hague, The Netherlands; "Department of Neurosurgery, Brain Center Rudolf Magnus University Medical Center Utrecht, Utrecht, The Netherlands; "Department of Neurosurgery, Haga Teaching Hospital, The Hague, The Netherlands

\section{Correspondence:}

Wouter A. Moojen, MD, PhD, MPH, Department of Neurosurgery, Haaglanden Medical Center, Lijnbaan 32 2512VA,

The Hague, The Netherlands.

E-mail:w.moojen@haaglandenmc.nl

Received, March 16, 2018

Accepted, September 12, 2018.

Copyright (C) 2018 by the

Congress of Neurological Surgeons

BACKGROUND: Long-term results from the International Subarachnoid Hemorrhage Trial (ISAT) and Barrow Ruptured Aneurysm Trial (BRAT) indicate considerably higher retreatment rates for aneurysms treated with coiling compared to clipping, but do not report the outcome of retreatment.

OBJECTIVE: To evaluate retreatment related outcomes.

METHODS: A meta-analysis in accordance with PRISMA guidelines was conducted using Medline search engines PubMed and EMBASE to identify articles describing outcomes after retreatment for intracranial aneurysms. Pooled prevalence rates for complete occlusion rate and mortality were calculated. Outcomes of different treatment and retreatment combinations were not compared because of indication bias.

RESULTS: Twenty-five articles that met the inclusion criteria were included in the metaanalysis. Surgery after coiling had a pooled complete occlusion rate of $91.2 \%$ (95\% confidence interval [Cl]: 87.0-94.1) and a pooled mortality rate of 5.6\% (95\% Cl: 3.7-8.3). Coiling after coiling had a pooled complete occlusion rate of $51.3 \%$ (95\% Cl: 22.1-78.0) and a pooled mortality rate of $0.8 \%(95 \% \mathrm{Cl}: 0.15-3.7)$. Surgery after surgery did not provide a pooled estimate for complete occlusion as only one study was identified but had a pooled mortality rate of $5.9 \%$ ( $95 \% \mathrm{Cl}: 3.1-11.2)$. Coiling after surgery had a pooled complete occlusion rate of $56.1 \%(95 \% \mathrm{Cl}$ : 11.4-92.7) and a pooled mortality rate of $9.3 \%$ (95\% Cl: 4.119.9). All pooled incidence rates were produced using random-effect models.

CONCLUSION: Surgical retreatment was associated with a high complete occlusion rate but considerable mortality. Conversely, endovascular retreatment was associated with low mortality but also a low complete occlusion rate.

KEY WORDS: Subarachnoid hemorrhage, Cerebral aneurysm, Retreatment, Meta-analysis, Coiling, Clipping

Neurosurgery 0:1-12, $2018 \quad$ DOI:10.1093/neuros/nyy455 www.neurosurgery-online.com

$\mathbf{T}$ he current mainstay treatment modalities for both ruptured and unruptured intracranial aneurysms are microsurgical clipping and endovascular treatment such as coiling. ${ }^{1,2}$ There is a growing preference to treat intracranial aneurysms with an endovascular treatment modality, as short-term and mediumlong-term outcomes seem to show a superi-

ABBREVIATIONS: CI, confidence interval; GOS, Glasgow Outcome Scale; ISAT, International Subarachnoid Hemorrhage Trial; mRS, modified Rankin Scale; NOS, Newcastle Ottawa Scale; PRISMA, Preferred Reporting Items for Systematic Reviews and Meta-Analyses

Supplemental digital content is available for this article at www.neurosurgery-online.com. ority over clipping with regard to morbidity, mortality, and functional outcomes. ${ }^{1-4}$ Endovascular treatment is also considered to be less invasive and is suggested to be preferred by most patients. ${ }^{1-4}$ However, regrowth and subsequent retreatment rate are considerably higher in patients treated with coiling compared to clipping in the long term (BRAT 6-yr followup data: retreatment: $16.4 \%$ for coiling vs $4.6 \%$ for clipping, respectively). ${ }^{2}$ Furthermore, the results from the International Subarachnoid Hemorrhage Trial (ISAT) indicate significantly higher rates of rebleeding and retreatment rates for endovascular-treated aneurysms compared to surgically treated aneurysms at $10-y r$ followup (retreatment: $17.4 \%$ for coiling vs $3.9 \%$ for clipping, respectively). ${ }^{1,5}$ However, there was no significant difference in mortality and 


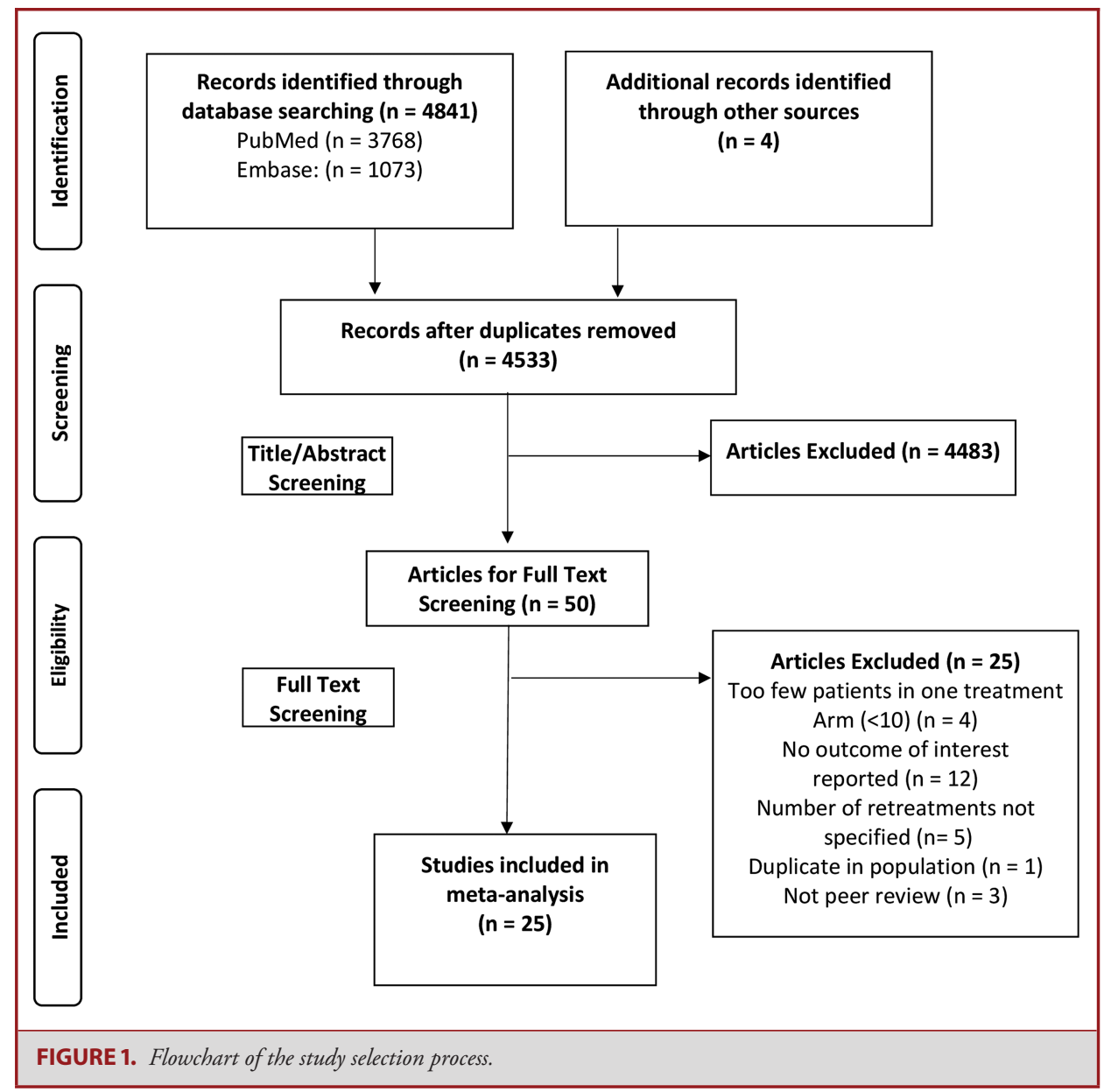

functional outcome, which may indicate that regrowth does not result in worse outcomes. ${ }^{1,5}$ Therefore, questions remain regarding possibility, efficacy, and safety of retreatment after regrowth as a result of the often-occurring regrowth of aneurysms. For instance, aneurysms may be difficult to surgically retreat because of increased mass, scar tissue, and may even require a bypass. $^{6}$

One meta-analysis indicated that microsurgical retreatment may be considered safe. ${ }^{7}$ However, this meta-analysis was limited by the studies included. Furthermore, other treatment and retreatment combinations were subjected to a meta-analysis. ${ }^{7}$ In addition, different treatment and retreatment combinations have not been compared. There is a great variety of initial treatment options which include: clipping, coiling, Pipeline Embolization Device (PED, $\odot$ Chestnut Medical, Medtronic Inc, Dublin, Ireland), surgical (high-flow) bypass, wrapping, and trapping, Woven Endobridge (WEB, oSequent Medical Inc, MicroVention Inc, Aliso Viejo, California) device, all of which can be used for retreatment. ${ }^{6,8-12}$ The aim of this meta-analysis was to evaluate the efficacy and safety of retreatment of intracranial aneurysms for all available treatment-retreatment combinations.

\section{METHODS}

\section{Study Selection}

A systematic review and meta-analysis of available literature was performed in accordance with the Preferred Reporting Items for Systematic Reviews and Meta-Analyses (PRISMA) guidelines. ${ }^{13}$ PubMed and Embase databases were searched through October 2017 for studies reporting outcomes of re-intervention for intracranial aneurysms (Figure 1). The search syntax was drafted with Appropriate Medical Subject Headings (MeSH) and Emtree terms for PubMed and Embase, respectively (Table, Supplemental Digital Content 1). The search was not limited by date of publication. Duplicates in identified articles were removed using Endnote X7.5 (Clarivate Analytics, Philadelphia, Pennsylvania). All abstracts were screened in duplicate and discrepancies between reviewers were resolved by a senior author. Articles that were selected for full-text review were evaluated by two authors. Reference lists were checked for possible additional articles. 


\section{Inclusion Criteria}

All studies that were screened full-text were included if the following inclusion criteria were met: (1) the study was original and written in English or Dutch, (2) the study included a treatment retreatment combination arm that consisted of a minimum of 10 patients, (3) the study was conducted in an adult population, (4) the study had been subjected to peer-review, and (5) the study-reported outcomes of retreatment for intracranial aneurysms. Only the most recent study was included if results from a series of patients had been reported in multiple articles.

\section{Data Extraction and Study Quality Assessment}

All included studies were evaluated for the following study characteristics: continent, year of publication, sample size, study design, and follow-up (months). The following population characteristics were extracted for the initial treatment and retreatment combinations: mean age (years), number of females and males, initial presentation (subarachnoid hemorrhage or incidental), initial treatment modality, retreatment modality, indication for initial treatment, indication for retreatment, aneurysm location, percentage of initial successful occlusion, mean total aneurysm size at retreatment, number of large aneurysms $(1.0-2.5 \mathrm{~cm})$, number of giant aneurysms $(>2.5 \mathrm{~cm})$, and mean time to retreatment (months). The following outcomes were extracted: percentage of complete occlusion on imaging (angiogram or computed tomography angiography), number of patients that died within $30 \mathrm{~d}$ after surgery (regardless of reason), and clinical outcome (modified Rankin Scale [mRS] or Glasgow Outcome Scale [GOS]). ${ }^{14,15}$ Clinical outcomes were only extracted if the patients' status before and after the retreatment procedure was documented and had less than $10 \%$ missing values. A good clinical outcomes score was defined as less than 3 and greater than 4 on the mRS and GOS, respectively. ${ }^{14,15}$ The extraction of the study characteristics and outcomes was performed by two independent investigators and discrepancies were solved by discussion or consultation of senior authors. All included studies were also evaluated for study quality using the Newcastle Ottawa Scale (NOS). ${ }^{16}$ Comparability was not scored for studies that did not have a comparison group. Again, discrepancies were solved by consultation of senior authors.

\section{Meta-Analysis}

The meta-analysis was conducted using R 3.4.2 (The R Foundation, Vienna, Austria) with use of the "meta" package, which is partially built on the "Metafor" package. ${ }^{17,18}$ Pooled prevalence ratios of complete occlusion rate and mortality rate were calculated using both fixedand random-effect models for the following treatment and re-treatment combinations: surgery after coiling, coiling after coiling, coiling after surgery, surgery after surgery, PED (Medtronic) after coiling, and PED after PED. Forest plots were created for the outcomes complete occlusion rate and mortality rate. The different initial treatment and retreatment combinations were not directly compared because of indication bias. The Cochran's $\mathrm{Q}$ test $(P<.10)$ and $\mathrm{I}^{2}$ statistic $\left(\mathrm{I}^{2}\right.$ value $>50 \%$ was considered significant) were used to evaluate possible heterogeneity among the included studies. ${ }^{19}$ Meta-regression was applied to identify sources of heterogeneity for the following study characteristics: continent, year of publication, NOS-score, percentage of females, mean age (years), percentage of patients that initially presented with a subarachnoid hemorrhage, percentage of initial successful treatment, mean total aneurysm size at retreatment, percentage of patients with an aneurysm located in the posterior circulation, percentage of patients with large or giant aneurysms, and mean time to re-intervention (months). Meta-regression separately was applied to all study characteristics and was only possible if a minimal of 3 studies were included in a specific treatment retreatment analysis and if no missing values were among the study characteristics and Bonferroni correction was applied to correct for multiple testing (critical $P$-value: .004 based on 13 degrees of freedom). Publication bias was evaluated by constructing Funnel plots, Eggers's linear regression test, and the Begg's and Mazumdar rank correlation test $(P$-value $<.05$ was considered significant $) .{ }^{20,21}$

\section{RESULTS}

The search strategy yielded 4529 studies after removal of duplicates (Figure 1). Twenty-five unique studies were included in the meta-analysis to evaluate outcomes of first retreatment for intracerebral aneurysms. ${ }^{5,6,9-12,22-40}$

\section{Baseline Characteristics}

The 25 studies reported the outcomes in 1064 patients (Table 1). The median of mean age of the populations was $50.6 \mathrm{yr}$ at time of retreatment and the median of mean female percentage of the populations was 65.0. The studies had a mean followup of 22.9 mo. Regarding aneurysm characteristics, the median of mean percentage of patients that initially presented with a subarachnoid hemorrhage was $75.9 \%$. The median of mean percentage of posterior location was $10.3 \%$. The median of mean size at retreatment was $7.0 \mathrm{~mm}$, median of mean percentage of large aneurysms was $18.3 \%$, and the median of mean percentage of giant aneurysms was $4.4 \%$. Regarding the retreatment, the median of mean number of months to retreatment was 20.8. Early retreatment (within the first month) occurred in $6.0 \%$ of cases (median of mean percentage). The NOS score did not vary greatly among the studies as most were retrospective case series and cohort studies that did not make comparison between the different treatment retreatment combinations, except for the ISAT. $^{5}$

Thirteen studies evaluated outcomes of surgery after coiling, ${ }^{6,11,22,24,28,29,31,33,34,36,38-40} 3$ studies evaluated outcomes of coiling after coiling, ${ }^{6,26,37} 4$ studies evaluated coiling after surgery, ${ }^{9,27,32,35} 3$ studies evaluated surgery after surgery, ${ }^{9,12,34} 2$ studied evaluated PED after coiling, ${ }^{23,30}$ and 2 studies evaluated PED after PED (Medtronic Inc). ${ }^{10,25}$

In studies that described outcomes of surgery after coiling, extrusion of coils and coil compaction were observed in $18.8 \%$ and $30.5 \%$ of cases, respectively. Coils were extracted in $22.7 \%$ of cases and intraoperative rupture occurred in $5.6 \%$ of cases. Clipping was performed in $89.2 \%$ of cases, a bypass procedure was performed in $4.6 \%$ of cases, wrapping in $4.3 \%$ of cases, trapping in $3.6 \%$ of cases, and parent artery occlusion in $1.4 \%$ of cases.

\section{Complete Occlusion}

Complete occlusion rates varied considerably among the treatment retreatment combinations (Table 2). Pooled 


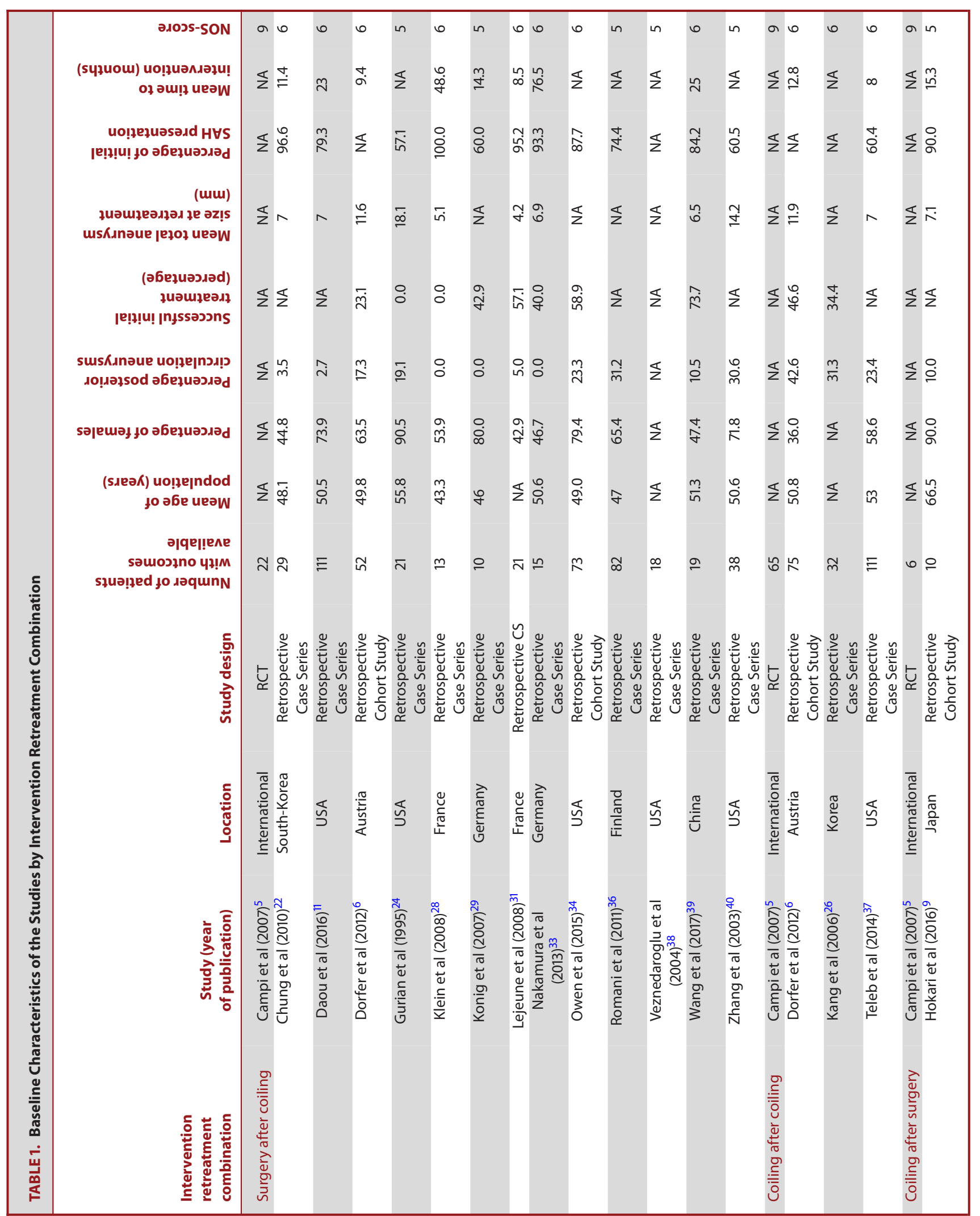




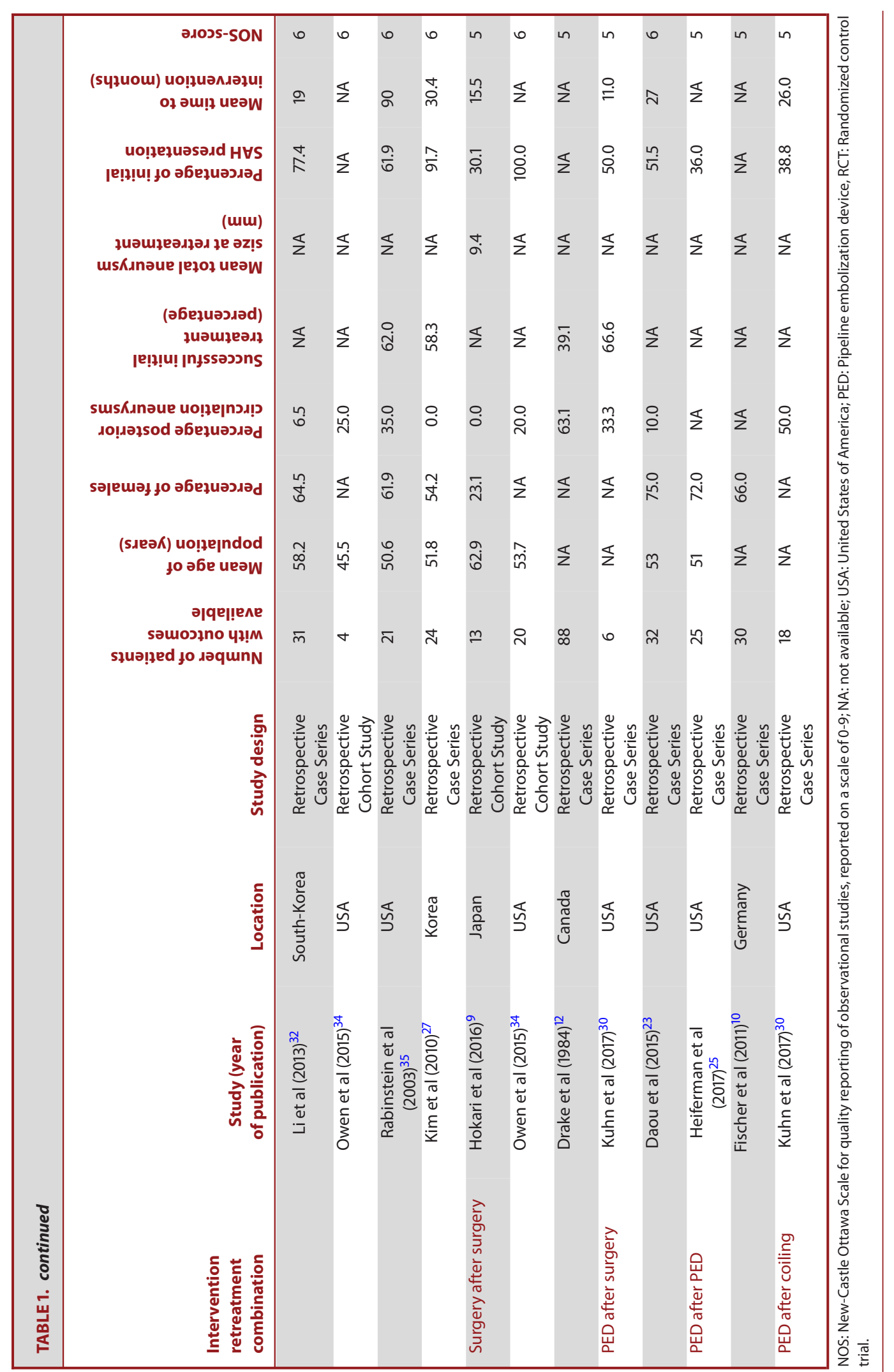




\begin{tabular}{|c|c|c|c|c|c|c|c|}
\hline \multirow{2}{*}{$\begin{array}{l}\text { Intervention } \\
\text { retreatment } \\
\text { combination }\end{array}$} & \multirow{2}{*}{ Study } & \multirow{2}{*}{$\begin{array}{l}\text { Complete } \\
\text { occlusion } \\
(\mathrm{n} / \mathrm{N}(\%))\end{array}$} & \multirow{2}{*}{$\begin{array}{l}\text { Mortality } \\
\text { occlusion } \\
(\mathrm{n} / \mathrm{N}(\%))\end{array}$} & \multicolumn{4}{|c|}{ Clinical outcome $^{a}$} \\
\hline & & & & \multicolumn{2}{|c|}{ Before treatment } & \multicolumn{2}{|c|}{ After treatment } \\
\hline \multirow{8}{*}{ Surgery after Coiling } & Campi et al $(2007)^{5}$ & NA & NA & $19 / 22$ & $3 / 21$ & $17 / 22$ & $5 / 22$ \\
\hline & Chung et al $(2010)^{22}$ & NA & $0 / 29(0.0)$ & $18 / 22$ & $4 / 22$ & $21 / 22$ & $1 / 22$ \\
\hline & Gurian et al $(1995)^{24}$ & NA & $3 / 21(14.3)$ & NA & NA & NA & NA \\
\hline & Klein et al $(2008)^{28}$ & 13/13 (100.0) & $0 / 13(0.0)$ & NA & NA & NA & NA \\
\hline & Konig et al $(2007)^{29}$ & $10 / 10(100.0)$ & $0 / 10(0.0)$ & NA & NA & NA & NA \\
\hline & Lejeune et al $(2008)^{31}$ & 19/21 (90.5) & $0 / 21(0.0)$ & $21 / 21$ & $0 / 21$ & $19 / 21$ & $2 / 21$ \\
\hline & Nakamura et al $(2013)^{33}$ & $15 / 15(100.0)$ & $1 / 15(6.7)$ & NA & NA & NA & NA \\
\hline & Owen et al $(2015)^{34}$ & 65/73 (89.0) & $3 / 73(4.1)$ & NA & NA & NA & NA \\
\hline \multirow{3}{*}{ Coiling after Coiling } & Dorfer et al $(2012)^{6}$ & $50 / 75(66.7)$ & $0 / 75(0.0)$ & NA & NA & NA & NA \\
\hline & Kang et al $(2006)^{26}$ & $11 / 32(34.4)$ & $0 / 32(0.0)$ & NA & NA & NA & NA \\
\hline & Teleb et al $(2014)^{37}$ & NA & $0 / 111(0.0)$ & NA & NA & NA & NA \\
\hline \multirow[t]{6}{*}{ Coiling after surgery } & Campi et al $(2007)^{5}$ & NA & NA & $6 / 6$ & $0 / 6$ & $4 / 6$ & $1 / 6$ \\
\hline & Hokari et al $(2016)^{9}$ & NA & $0 / 10(0.0)$ & $9 / 10$ & $1 / 10$ & $8 / 10$ & $2 / 10$ \\
\hline & Li et al $(2013)^{32}$ & 9/31 (29.0) & $2 / 31(6.5)$ & NA & NA & NA & NA \\
\hline & Owen et al $(2015)^{34}$ & NA & $0 / 4(0.0)$ & NA & NA & NA & NA \\
\hline & Rabinstein et al (2003) 35 & $17 / 21(81.0)$ & $3 / 21(14.3)$ & NA & NA & NA & NA \\
\hline & Kim et al $(2010)^{27}$ & NA & NA & $19 / 19$ & $0 / 19$ & $16 / 19$ & $3 / 19$ \\
\hline \multirow[t]{3}{*}{ Surgery after surgery } & Hokari et al (2016) ${ }^{9}$ & NA & $0 / 13(0.0)$ & $12 / 13$ & $1 / 13$ & $10 / 13$ & $3 / 13$ \\
\hline & Owen et al $(2015)^{34}$ & NA & $2 / 20(10.0)$ & NA & NA & NA & NA \\
\hline & Drake et al $(1984)^{12}$ & $102 / 115$ (88.7) & $6 / 115(5.2)$ & NA & NA & NA & NA \\
\hline
\end{tabular}

PED: Pipeline embolization device; NA: not available.

${ }^{\mathrm{a}} \mathrm{Good}$ outcome was defined as $\mathrm{mRS}<3$ or a GOS $>4$.

prevalence rates for complete occlusion were $91.2 \%(95 \%$ confidence interval [CI]: 87.0-94.2) for surgery after coiling, 51.3\% (95\% CI: 22.1-78.0) for coiling after coiling, $72.1 \%$ (95\% CI: 57.0- 83.3) for PED after coiling, 56.1\% (95\% CI: 11.4-92.7) for coiling after surgery, and 58.2\% (95\% CI: 39.075.2) for PED after PED, respectively (Random-effect model, Figure 2, Table, Supplemental Digital Content 2). Fixed-effect models yielded similar results. It was not possible to calculate a prevalence rate for surgery after surgery as only one study was available for analysis, which reported a complete occlusion rate of $89 \% .^{12}$

The treatment retreatment combinations coiling after coiling and coiling after surgery came with considerable heterogeneity $(88.9 \%$ and $91.5 \%$, respectively). No significant sources of heterogeneity were identified for surgery after coiling using meta- regression after correction for multiple testing. Meta-regression for other treatment and retreatment combination was not possible due to the low number of studies included in the analysis. It was only possible to evaluate publication bias in the surgery after coiling treatment retreatment combination, which was insignificant, as the other analysis had too few studies to evaluate publication bias.

\section{Mortality}

Similar to complete occlusion rates, the pooled prevalence rates of mortality varied considerably among the treatment retreatment combinations (Table 2). Pooled mortality rates for mortality were 5.6\% (95\% CI: 3.7-8.3) for surgery after coiling, $0.8 \%$ (95\% CI: 0.15-3.7) for coiling after coiling, 2.2\% (95\% CI: 0.3-14.3) for PED after coiling, 5.9\% (95\% CI: 3.1-11.2) for 


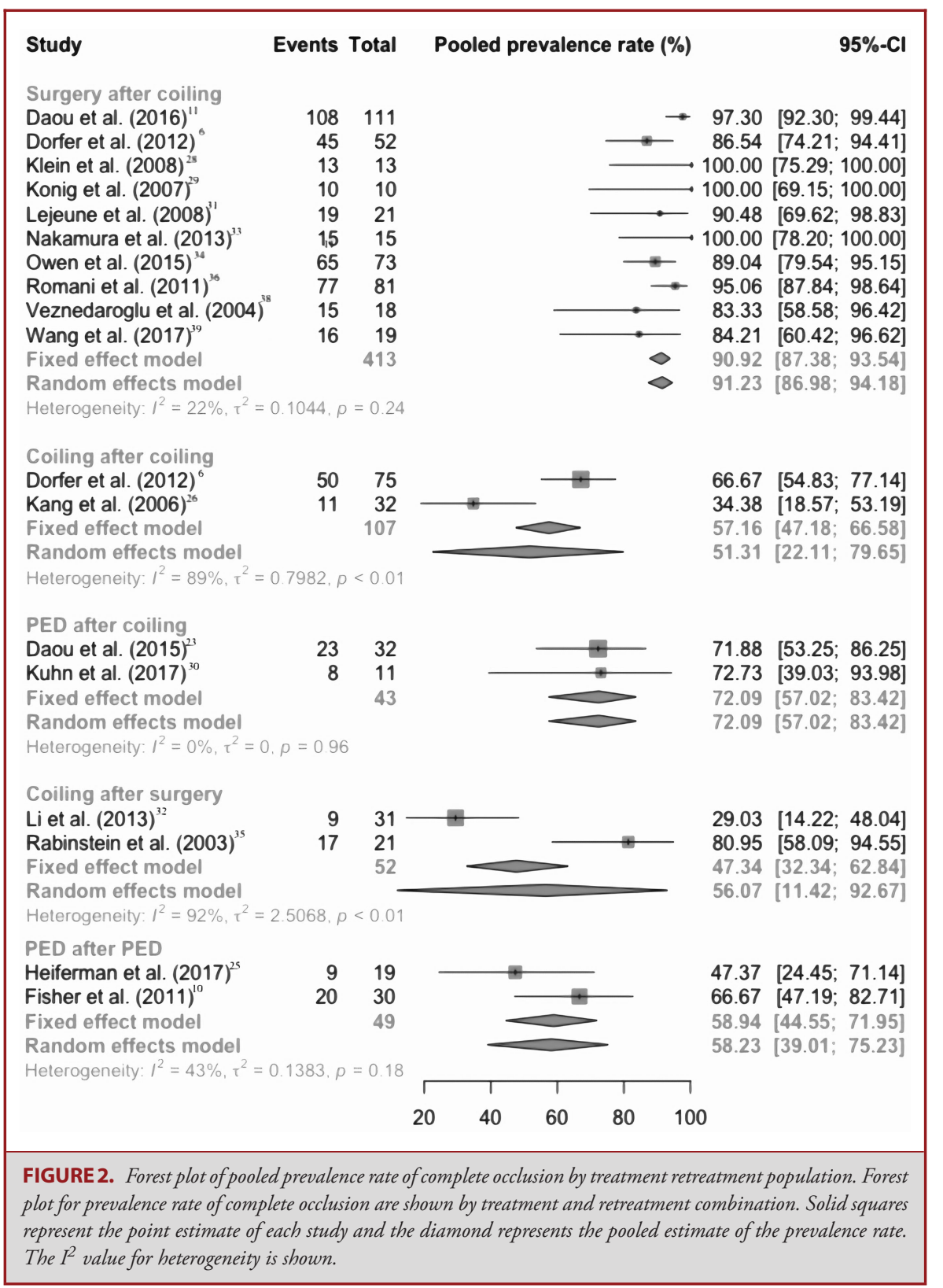

surgery after surgery, 9.3\% (95\% CI: 4.1-19.9) for coiling after surgery, and 2.0\% (95\% CI: 0.3-12.9) for PED after PED, respectively (Random-effect model, Figure 3, Table, Supplemental Digital Content 2). Fixed-effect models showed similar results. All the intervention retreatment combinations came with low heterogeneity $\left(\mathrm{I}^{2}=0.0 \%\right.$ for all studies). With regard to publication bias, the pooled prevalence rate of mortality for surgery after coiling may be subject to publication bias (Egger's test $P$-value: .08). However, the Funnel plot indicated no publication bias (not shown), the trim and fill method yielded a similar pooled prevalence rate (pooled prevalence rate: $0.8 \%, 95 \%$
CI: 0.2-3.7), and the Begg's test indicated no significant publication bias $(P=.12)$. It was not possible to evaluate publication bias for the surgery after surgery, PED after coiling, and PED after PED treatment retreatment combinations due to a low number of studies included in the analysis.

For surgery after coiling, only publication year was identified as a potential source of heterogeneity (estimate: $-0.06, P$-value: .04 ), but this association did not remain significant after correction for multiple testing. Meta-regression for other treatment retreatment combinations was not possible due to the low number of studies included in the analysis. 


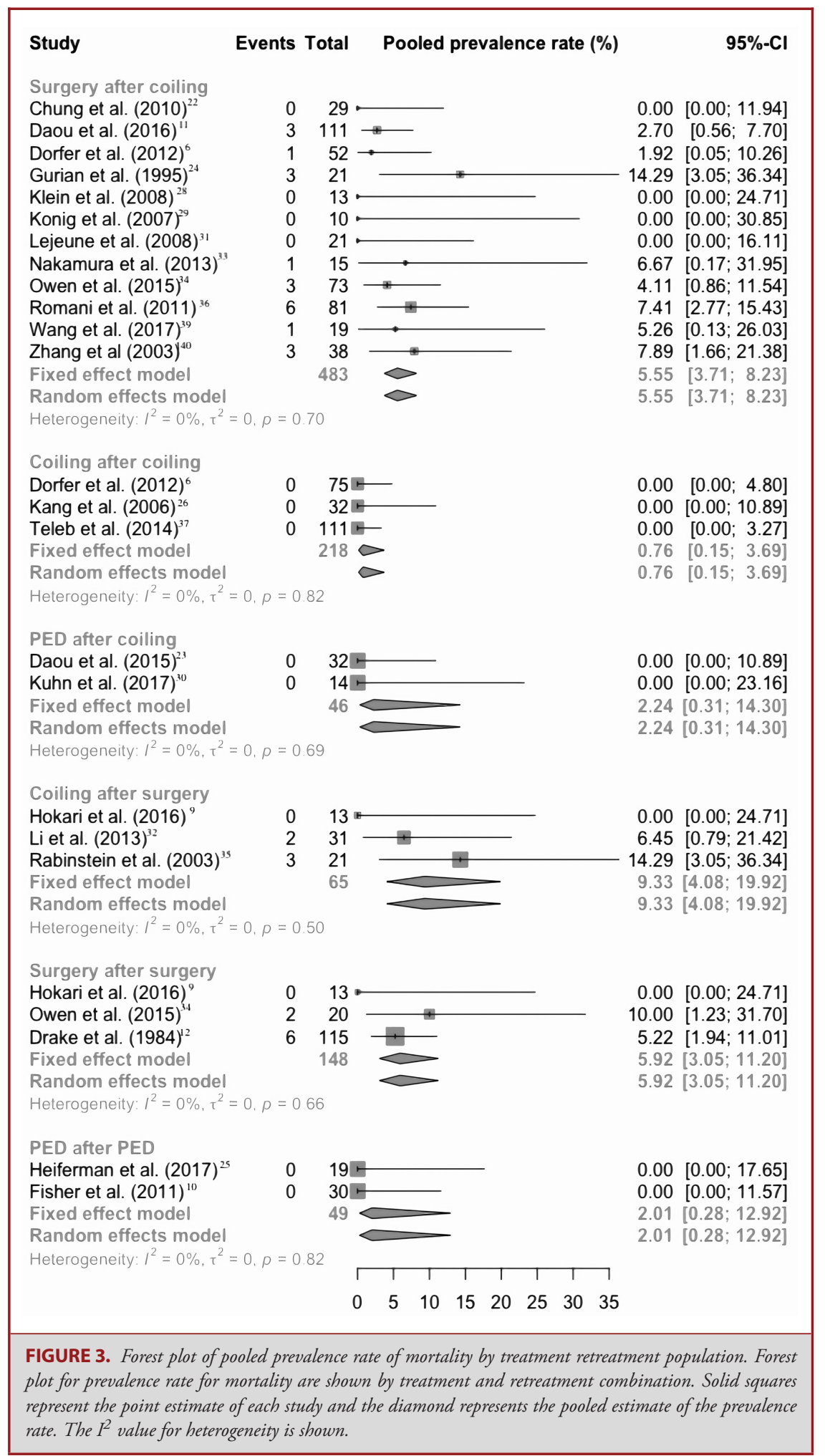




\section{Functional Outcomes}

Contrary to the occlusion rates or mortality rates, the functional outcomes were not reported consistently (Table 2). $., 9,10,22,27,30,31$ The clinical improvement or deterioration after the second intervention varied considerably among the treatment retreatment combinations. In general, no large differences were seen in functional outcome before and after intervention between the different treatment and retreatment combinations.

\section{DISCUSSION}

This meta-analysis aimed to evaluate outcomes of retreatment of intracranial aneurysms. Overall, aneurysms retreated with surgery showed a high rate of complete occlusion. However, this came with a relatively high pooled prevalence rate of mortality. Contrarily, intracranial aneurysms retreated with coiling or a PED (Medtronic) have a relative low complete occlusion rate, but also a low pooled prevalence rate of mortality. Functional outcomes were reported very infrequently and did not show a great variation between the different treatment retreatment combinations. The overall quality of the studies was low, as no prospective studies were available, except for the ISAT, which only reported functional outcomes. ${ }^{5}$

The occlusion rate for surgically retreated patients appears to be high based on the results of our meta-analysis. One other meta-analysis suggested an occlusion rate of $98.3 \%{ }^{7}$ One systematic review evaluating outcomes of surgically treated aneurysms concluded an occlusion rate of $93 \% .{ }^{8}$ These outcomes are broadly similar to the findings in this meta-analysis. One series that evaluated outcomes of 2360 intracranial aneurysm patients, initially treated with endovascular approaches, found that 350 $(12.3 \%)$ patients required endovascular retreatment and reported a complete occlusion rate of only $46.9 \%$ after the second session of coiling. ${ }^{41}$ Furthermore, 94 patients required three or more coiling sessions with a complete occlusion rate of $35.6 \%$ (maximum of 9 sessions, $\mathrm{n}=1$ ). ${ }^{41}$ The potential necessity of multiple subsequent recoiling sessions may emphasize the need from complete initial occlusion of aneurysms. The latter study was not included in this meta-analysis as the initial endovascular treatment was preceded by various microsurgical procedures in some patients. ${ }^{41}$ However, incomplete coiling is not the only factor associated with regrowth as increased total aneurysm size, packing density, older age, male sex, hypertension, and ruptured aneurysm have also been associated with aneurysm regrowth after coiling in retrospective analyses. ${ }^{42-44}$

The mortality rate seems to be high in patients that received surgical retreatment based on our meta-analysis. Another metaanalysis suggested that mortality may actually be 0\% (95\% $\mathrm{CI}=0.0 \%-2.5 \%) .^{7}$ One other review reported a mortality prevalence of $3.6 \%$ for aneurysms that were surgically retreated after initial endovascular occlusion, which is similar to the findings in this meta-analysis. ${ }^{8}$ One explanation for the mortality in surgi- cally retreated patients rate may be the necessity of trapping, wrapping, ligation, or a bypass, which was necessary in $1.9 \%$, $2.7 \%, 1.8 \%$, and $2.1 \%$ of cases, respectively, which is largely similar to the findings of this study. ${ }^{8}$ Another explanation may also be mortality due to non-procedural complications or presentation with a rebleed, as was seen in the cohort described by Romani et al. ${ }^{36}$ One large series investigating multiple recoiling sessions in 350 patients reported a morbidity rate of $2.2 \%$ and only had one mortality. ${ }^{41}$ Coiling after surgery was associated with a relatively high mortality, but this was probably the result of rebleed related complications in the two studies that had mortalities. ${ }^{32,35}$

The high mortality and poor occlusion rates for surgical retreatment and endovascular retreatment, respectively, show the importance of treatment modality selection when patients initially present with intracranial aneurysms. Initial coiling may be preferable because of lower morbidity and less invasive nature if no additional retreatment is to be expected. ${ }^{5}$ The necessity for retreatment after coiling is approximately three to four times higher than after microsurgical clipping based on two randomized control trials (RCTs). ${ }^{2,5}$ Therefore, patients that require surgery after coiling would probably not have needed a secondary procedure if surgery had been the primary treatment modality. Furthermore, recoiling may increase the size of the aneurysm with every additional placement of coils due to its low success rate. The difficulty of surgical treatment of previously coiled aneurysms probably increases after every recoiling session as the aneurysm increases in size. Similarly, the complete occlusion rate appears to go down with every subsequent recoiling procedure. ${ }^{41}$ Although this does not seem to result in mortality for patients, rebleeding rates could be higher and patients may develop symptoms as the result of mass-effect from the aneurysm. ${ }^{41,45,46}$ Currently, no prospective and comparative outcomes are known for retreated intracranial aneurysm and little is known on factors that contribute to regrowth.

This is the first meta-analysis that evaluated the outcomes of retreatment for intracranial aneurysms and was conducted in accordance with PRISMA guidelines. ${ }^{13}$ This meta-analysis also evaluated all available intervention and retreatment combinations. However, this meta-analysis is limited by several factors. The available studies from the literature were of poor quality, based on the NOS-scale. All studies were retrospective in nature and generally had a small sample size and limited follow-up. There was little consistency with regard to reported outcomes as most studies only consistently reported mortality and complete occlusion rate, which was why no meta-analysis was conducted for functional outcomes. The limited reporting on baseline characteristics and outcomes of specific subgroups such as patients that initially presented with a subarachnoid hemorrhage did not allow for further subgroup analyses. Authors were not contacted to provide the necessary information due to the great many studies that did not present this information. This especially holds serious implications for mortality as other unevaluated 
factors such as the number of patients presenting with a rebleed and nonprocedure related complications may influence mortality. The number of studies could also be considered low for the respective treatment retreatment combinations (eg only two studies reporting on outcomes of coiling after coiling were included). This is partially the result of the inclusion criteria of a minimum of 10 patients per arm and that only outcomes of first retreatment were evaluated. There was also considerable heterogeneity among various outcomes for which it was often not possible to identify contributing factors due to a low number of studies and variation in reporting of base-line characteristics by meta-regression. No comparison was made between the different treatment and retreatment combinations with regard to outcomes because of indication bias. It was also not possible to study the effect of timing of retreatment in relation to outcomes as timing was rarely reported. Meta-regression was also only applied for individual study characteristics and was often not possible due to the low number of studies and variation in reporting. Furthermore, none of the associations identified using meta-regression remained significant after correction for multiple testing. Findings of this meta-analysis were not validated in internal dataset with cross validation or in an external dataset as these were not available.

Knowledge of the outcomes of retreatment could be expanded by prospective evaluation of outcomes. A potential trial design could be a prospective registry that evaluates outcomes of intracranial aneurysms irrespective of initial treatment. This could both provide insight into which aneurysms require retreatment on the long term, how the necessity of retreatment can be avoided, and what retreatment strategy results in superior outcomes. The outcomes of such a registry could also prediction model to aid clinical decision-making and improve outcomes of intracranial aneurysm patients.

\section{CONCLUSION}

Surgical retreatment of intracranial aneurysms may be associated with relatively high occlusion rates but also a relatively high mortality. Contrarily, secondary coiling may be associated with relatively lower mortality but also with low rates of complete occlusion. Outcomes of this meta-analysis should be interpreted with caution due to various limitations. Nevertheless, the outcomes from this meta-analysis could potentially stress the need for complete initial treatment of intracranial aneurysms to prevent the retreatment. The findings of this meta-analysis could also potentially strengthen the argument for opting to clip an intracranial aneurysm when initial coiling may not result in complete occlusion. Further knowledge on what contributes to regrowth of coiled aneurysm is needed to optimize initial treatment selection for individual patients.

\section{Disclosure}

The authors have no personal, financial, or institutional interest in any of the drugs, materials, or devices described.

\section{REFERENCES}

1. Molyneux AJ, Birks J, Clarke A, Sneade M, Kerr RS. The durability of endovascular coiling versus neurosurgical clipping of ruptured cerebral aneurysms: 18 year follow-up of the UK cohort of the International Subarachnoid Aneurysm Trial (ISAT). Lancet. 2015;385(9969):691-697.

2. Spetzler RF, McDougall CG, Zabramski JM, et al. The barrow ruptured aneurysm trial: 6-year results. J Neurosurg. 2015;123(3):609-617.

3. Lawton MT, Vates GE. Subarachnoid hemorrhage. $N$ Engl J Med. 2017;377(3):257-266.

4. Spetzler RF, Zabramski JM, McDougall CG, et al. Analysis of saccular aneurysms in the barrow ruptured aneurysm trial. J Neurosurg. 2018;128(1):120-125.

5. Campi A, Ramzi N, Molyneux AJ, et al. Retreatment of ruptured cerebral aneurysms in patients randomized by coiling or clipping in the International Subarachnoid Aneurysm Trial (ISAT). Stroke. 2007;38(5):15381544 .

6. Dorfer C, Gruber A, Standhardt H, Bavinzski G, Knosp E. Management of residual and recurrent aneurysms after initial endovascular treatment. Neurosurgery. 2012;70(3):537-554; discussion 553-534

7. Petr O, Brinjikji W, Thome C, Lanzino G. Safety and efficacy of microsurgical treatment of previously coiled aneurysms: A systematic review and meta-analysis. Acta Neurochir. 2015;157(10):1623-1632.

8. Arnaout OM, El Ahmadieh TY, Zammar SG, et al. Microsurgical treatment of previously coiled intracranial aneurysms: systematic review of the literature. World Neurosurg. 2015;84(2):246-253.

9. Hokari M, Kazumara K, Nakayama N, et al. Treatment of recurrent intracranial aneurysms after clipping: a report of 23 cases and a review of the literature. World Neurosurg. 2016;92:434-444.

10. Fischer S, Vajda Z, Aguilar Perez M, et al. Pipeline embolization device (PED) for neurovascular reconstruction: Initial experience in the treatment of 101 intracranial aneurysms and dissections. Neuroradiology. 2012;54(4):369-382.

11. Daou B, Chalouhi N, Starke RM, et al. Clipping of previously coiled cerebral aneurysms: Efficacy, safety, and predictors in a cohort of 111 patients. J Neurosurg. 2016;125(6):1337-1343.

12. Drake CG, Friedman AH, Peerless SJ. Failed aneurysm surgery. J Neurosurg. 1984;61(5):848-856.

13. Moher D, Liberati A, Tetzlaff J, Altman DG, Group P. Preferred reporting items for systematic reviews and meta-analyses: The PRISMA statement. Ann Intern Med. 2009;151(4):264-269, W264.

14. Jennett B, Bond M. Assessment of outcome after severe brain damage. Lancet. 1975;1(7905):480-484.

15. Wilson JT, Hareendran A, Grant M, et al. Improving the assessment of outcomes in stroke: Use of a structured interview to assign grades on the modified rankin scale. Stroke. 2002;33(9):2243-2246.

16. Wells GA, Shea B, O'Connell D, et al. The Newcastle-Ottawa Scale (NOS) for assessing the quality of nonrandomised studies in meta-analyses. 1999; http://www. ohri.ca/programs/clinical_epidemiology/oxford.asp. Accessed June 62017.

17. Viechtbauer W. Conducting meta-analyses in $\mathrm{R}$ with the metafor Package. J Stat Soft. 2010;36(3):1-48

18. Schwarzer G. meta: An R package for meta-analysis. R News. 2007;7(3):40-45.

19. Higgins JP, Thompson SG, Deeks JJ, Altman DG. Measuring inconsistency in meta-analyses. BMJ. 2003;327(7414):557-560.

20. Begg CB, Mazumdar M. Operating characteristics of a rank correlation test for publication bias. Biometrics. 1994;50(4):1088-1101.

21. Egger M, Davey Smith G, Schneider M, Minder C. Bias in meta-analysis detected by a simple, graphical test. BMJ. 1997;315(7109):629-634.

22. Chung J, Lim YC, Kim BS, Lee D, Lee KS, Shin YS. Early and late microsurgical clipping for initially coiled intracranial aneurysms. Neuroradiology. 2010;52(12):1143-1151.

23. Daou B, Starke RM, Chalouhi N, et al. The Use of the pipeline embolization device in the management of recurrent previously coiled cerebral aneurysms. Neurosurgery. 2015;77(5):692-697; discission 697.

24. Gurian JH, Martin NA, King WA, Duckwiler GR, Guglielmi G, Vinuela F. Neurosurgical management of cerebral aneurysms following unsuccessful or incomplete endovascular embolization. J Neurosurg. 1995;83(5):843853.

25. Heiferman DM, Billingsley JT, Kasliwal MK, et al. Use of flow-diverting stents as salvage treatment following failed stent-assisted embolization of intracranial aneurysms. J Neurointervent Surg. 2016;8(7):692-695. 
26. Kang HS, Han MH, Kwon BJ, Kwon OK, Kim SH. Repeat endovascular treatment in post-embolization recurrent intracranial aneurysms. Neurosurgery. 2006;58(1):60-70; discussion 60-70.

27. Kim BM, Kim DJ, Kim DI, Park SI, Suh SH, Won YS. Clinical presentation and outcomes of coil embolization of remnant or recurred intracranial aneurysm after clipping. Neurosurgery. 2010;66(6):1128-1133; discussion 1133.

28. Klein O, Colnat-Coulbois S, Civit T, et al. Aneurysm clipping after endovascular treatment with coils: A report of 13 cases. Neurosurg Rev. 2008;31(4):403-411; discussion 410-401.

29. Konig RW, Kretschmer T, Antoniadis G, et al. Neurosurgical management of previously coiled recurrent intracranial aneurysms. Zentralbl Neurochir. 2007;68(1):8-13.

30. Kuhn AL, de Macedo Rodrigues K, Lozano JD, et al. Use of the Pipeline embolization device for recurrent and residual cerebral aneurysms: A safety and efficacy analysis with short-term follow-up. J Neurointervent Surg. 2017;9(12):1208-1213.

31. Lejeune JP, Thines L, Taschner C, Bourgeois P, Henon H, Leclerc X. Neurosurgical treatment for aneurysm remnants or recurrences after coil occlusion. Neurosurgery. 2008;63(4):684-692; discussion 691-682.

32. Li K, Cho YD, Kang HS, Kim JE, Han MH, Lee YM. Endovascular management for retreatment of postsurgical intracranial aneurysms. Neuroradiology. 2013;55(11):1345-1353

33. Nakamura M, Montibeller GR, Gotz F, Krauss JK. Microsurgical clipping of previously coiled intracranial aneurysms. Clin Neurol Neurosurg. 2013;115(8):1343-1349.

34. Owen CM, Montemurro N, Lawton MT. Microsurgical Management of Residual and Recurrent Aneurysms After Coiling and Clipping. Neurosurgery. 2015;62(suppl 1):92-102.

35. Rabinstein AA, Nichols DA. Endovascular coil embolization of cerebral aneurysm remnants after incomplete surgical obliteration. Stroke. 2002;33(7):1809-1815.

36. Romani R, Lehto H, Laakso A, et al. Microsurgery for previously coiled aneurysms: experience with 81 patients. Neurosurgery. 2011;68(1):140-154; discussion 153-144

37. Teleb MS, Pandya DJ, Castonguay AC, et al. Safety and predictors of aneurysm retreatment for remnant intracranial aneurysm after initial endovascular embolization. J Neurointervent Surg. 2014;6(7):490-494.

38. Veznedaroglu E, Benitez RP, Rosenwasser RH. Surgically treated aneurysms previously coiled: lessons learned. Neurosurgery. 2004;54(2):300-305.

39. Wang HW, Sun ZH, Wu C, Xue Z, Yu XG. Surgical management of recurrent aneurysms after coiling treatment. Br J Neurosurg. 2017;31(1):96-100.

40. Zhang YJ, Barrow DL, Cawley CM, Dion JE. Neurosurgical management of intracranial aneurysms previously treated with endovascular therapy. Neurosurgery. 2003;52(2):283-295.

41. Henkes H, Fischer S, Liebig T, et al. Repeated endovascular coil occlusion in 350 of 2759 intracranial aneurysms: Safety and effectiveness aspects. Neurosurgery. 2008;62(6 suppl 3):1532-1537.

42. Lv N, Zhao R, Yang $P$, et al. Predictors of recurrence after stent-assisted coil embolization of paraclinoid aneurysms. J Clin Neurosci. 2016;33:173-176.

43. dos Santos MP, Sabri A, Dowlatshahi D, et al. Survival analysis of risk factors for major recurrence of intracranial aneurysms after coiling. Can J Neurol Sci. 2015;42(01):40-47.

44. Mascitelli JR, Oermann EK, De Leacy RA, Moyle H, Mocco J, Patel AB. Predictors of treatment failure following coil embolization of intracranial aneurysms. J Clin Neurosci. 2015;22(8):1275-1281.

45. Kawabata S, Toyota S, Kumagai T, Goto T, Mori K, Taki T. Direct surgery of previously coiled large internal carotid ophthalmic aneurysm for the purpose of optic nerve decompression. J Neurol Surg Rep. 2017;78(01):e20-e25.

46. van Eijck M, Bechan RS, Sluzewski M, Peluso JP, Roks G, van Rooij WJ. Clinical and imaging follow-up of patients with coiled basilar tip aneurysms up to 20 years. AJNR Am J Neuroradiol. 2015;36(11):2108-2113.

Supplemental digital content is available for this article at www. neurosurgery-online.com.

Supplemental Digital Content 1. Table. Search syntax.

Supplemental Digital Content 2. Table. Outcomes of meta-analysis by outcome.

\section{Acknowledgment}

The authors would like to thank Pauline Wiersma (librarian at Utrecht University Medical Center/Utrecht University) who helped designing the search strategy.

\section{COMMENTS}

$\mathrm{n}$ this present meta-analysis, the authors amassed a cohort of

1064 patients with previously treated aneurysms who consequently underwent retreatment with a variety of surgical and endovascular modalities. As might be expected, microsurgical treatment was found to have high rates of aneurysm occlusion $(91 \%$ and $89 \%$ after previous coiling and surgery, respectively). However, retreatment with surgery was associated with higher periprocedural mortality, compared to endovascular techniques. In contrast, meta-analysis of endovascular retreatment modalities illustrated high heterogeneity in results, with occlusion rates varying greatly between individual studies and also between retreatment categories (ie, coiling versus flow-diversion).

Using a broad swath of published literature, this meta-analysis confirms the inherent risks of both surgical and endovascular aneurysm retreatment. Furthermore, as the previously established standard, microsurgical techniques are relatively consistent in providing good overall occlusion rates after retreatment. In contrast, endovascular therapy (both coiling and flow-diversion) represents a more contemporary retreatment option, and thus may have lower occlusion rates and a wider variance in success given the inherent learning curve.

While this study incorporates a large patient population with a relatively rare pathology, the overall meta-analysis should be considered in light of its numerous limitations. First, the individual studies included in analysis are highly heterogeneous in methodology, results, and outcomes, such that only occlusion rates and mortality could be assessed. Second, the majority of studies were either retrospective case series or cohort analysis, both of which may be methodologically biased. Lastly, in most comparison categories two or fewer studies were cumulatively assessed (coiling after coiling, PED [Medtronic] after coiling, coiling after surgery, surgery after surgery, and PED after PED), again perhaps biasing the overall statistical analysis.

Nonetheless, limitations considered, this manuscript represents a valuable assessment of an otherwise important debate within the neurosurgical field. Furthermore, it suggests the need for more high quality evidence in aneurysm retreatment strategies.

\section{Kurt Yaeger \\ J. Mocco \\ New York, New York}

A lthough previous prospective studies have reported a significantly higher need for retreatment of aneurysms after coiling when compared to microsurgical clipping, ${ }^{1,2}$ the specific outcomes of these retreatment procedures are rarely reported. In this paper, the authors present a meta-analysis of the literature specifically regarding outcomes of aneurysm retreatment. Twenty-five articles reporting outcomes in 1064 patients were included with results being categorized based on both initial treatment and retreatment modality. The highest pooled rate of complete occlusion was found with surgery after coiling $(91.2 \%)$, and lower pooled complete occlusion rates were evident for coiling after coiling $(51.3 \%)$, coiling after surgery $(56.1 \%)$, PED (Medtronic) after coiling $(72.1 \%)$, and PED after PED (58.2\%). No pooled analysis was performed for surgery after surgery, as only one study was included, with an occlusion 
rate of $89 \%$. Although surgery after prior coiling was found to have the highest rate of subsequent complete occlusion, it was also found to have a relatively high pooled mortality rate of $5.6 \%$. Additional pooled mortality rates were $0.8 \%$ for coiling after coiling, $2.2 \%$ for PED after coiling, $2.0 \%$ for PED after PED, $5.9 \%$ for surgery after surgery, and 9.3\% for coiling after surgery. Functional outcomes were not reported consistently within the selected studies and therefore were not included in the meta-analysis.

Selecting a secondary treatment modality for a previously treated aneurysm is a complex process based on numerous radiographic and clinical characteristics (eg, aneurysm size, aneurysm location, remnant morphology, patient age, patient comorbidities, etc). In this study, outcomes were reported separately with regard to initial treatment and retreatment modality, and thus serve as a useful summary of existing literature. However, it is important to note that these subgroups cannot be directly compared in terms of outcome given the inherent indication bias. Nonetheless, a few concepts seem to be generally supported by the data:

1. Similar to the outcomes after initial clipping, surgery as a treatment modality results in high occlusion rates at time of re-treatment.
2. Despite the imperfect occlusion rates with endovascular retreatment modality, the risk in terms of mortality is generally low, although interestingly the highest mortality rate was seen in the coiling after surgery group; the authors surmise that this is likely related to aneurysmal rebleeding rather than procedure-related complications.

3. Retreatment is not without risk, and therefore aneurysm recurrence and retreatment should routinely be included as a relevant endpoint in outcome studies evaluating new aneurysm treatment modalities or comparing the efficacy of different modalities.

Ashley Barks

Sepideh Amin-Hanjani Chicago, Illinois

1. Molyneux AJ, Birks J, Clarke A, Sneade M, Kerr RS, et al. The durability of endovascular coiling versus neurosurgical clipping of ruptured cerebral aneurysms: 18 year follow-up of the UK cohort of the International Subarachnoid Aneurysm Trial (ISAT). Lancet. 2015;385(9969):691-697.

2. Spetzler RF, McDougall CG, Zabramski JM, et al.. The Barrow Ruptured Aneurysm Trial: 6-year results. J Neurosurg. 2015;123(3):609-617. 